\title{
Detection and management of eating disorders by general practitioners in regional Australia
}

\author{
Candice P Boyd ${ }^{1}$, Damon L Aisbett ${ }^{1}$, Andrew Howard ${ }^{2}$ and Toula Filiades ${ }^{3}$ \\ 1. Rural Adolescent Mental Health Group, Centre for Health Research and Practice, University \\ of Ballarat, Victoria, Australia \\ 2. Ballarat and District Division of General Practice, Ballarat, Victoria, Australia \\ 3. Centre of Excellence in Eating Disorders, Royal Melbourne Hospital, Parkville, Australia
}

\begin{abstract}
The aim of this study was to explore the prevalence of eating disorders in primary care in the Ballarat region and to highlight the role of GPs in the detection and management of eating disorders in regional Australia. We used anonymous data previously collated by the Ballarat and District Division of General Practice on the prevalence of eating disorders and patterns of referral of eating disorder patients among GPs in their Division. Over half of GPs surveyed indicated that they treat patients with eating disorders within their practice rather than referring patients to other services. In referring on, GPs were more likely to refer to mental health professionals and dietitians. A notable finding was that these regional GPs were more likely to refer to metropolitan specialist services than local hospitals if their patients required an admission. GPs in regional Australia do significant work to detect and manage patients with clinical eating disorders in the absence of locally-based, specialist services. In this context, we recommend the establishment of linkage partnerships between GPs and mental health practitioners to facilitate early intervention for rural and regional eating disorder patients. Further research into the current treatment practices of regional GPs is also needed to ascertain their specific training needs with respect to this patient population.
\end{abstract}

\section{Keywords}

eating disorders, general practitioners, primary health care, referral, detection, early intervention

\section{Introduction}

Eating disorders have high rates of chronicity and morbidity making them amongst the most challenging mental disorders to treat (Steinhausen, Rauss-Mason \& Seidel, 2003). The length of time between onset of an eating disorder and its detection and subsequent diagnosis is an indicator of poor prognosis (Hall,
Slim \& Hawker, 1984). Early detection is more likely to occur in primary care settings as eating disorder patients often present to general practitioners (GPs) with gynaecological or gastrointestinal complaints or concerns about weight loss before they present with psychological symptoms (Hay, Marley \& Lemar, 1998; Ogg, Millar \& Pusztai, 1997).

Contact: $\quad$ Candice Boyd, Rural Adolescent Mental Health Group, Centre for Health Research and Practice, University of Ballarat, University Drive, Ballarat, Victoria, 3350. c.boyd@ballarat.edu.au.

Citation: $\quad$ Boyd, C.P., Aisbett, D.L., Howard, A., \& Filiades, T. (2007). Detection and management of eating disorders by general practitioners in regional Australia. Australian e-Journal for the Advancement of Mental Health, 6(2), www.auseinet.com/journal/vol6iss2/boyd.pdf

Published by: $\quad$ Australian Network for Promotion, Prevention and Early Intervention for Mental Health (Auseinet) www.auseinet.com/journal

Received 13 December 2006; Revised 27 April 2007; Accepted 27 April 2007 
The role of the GP in the management of eating disorders lies primarily in monitoring and treating the medical complications of the disorder, as well as educating patients about the potential effects of these (Mehler, 2001). The needs of patients with clinical eating disorders anorexia nervosa and bulimia nervosa - are high and necessitate a team approach. There is an important role for GPs, particularly those in rural and regional areas, as the coordinator of a multidisciplinary team which would typically comprise the GP, a dietitian, and a therapist working in collaboration (Sayag \& Latzer, 2002). In less severe cases of eating disorder, the GP may elect to be the sole provider of psychological treatment. Guided self-help approaches for the treatment of bulimia nervosa in primary care by GPs have been shown to be highly efficacious in randomised controlled trials (Banasiak, Paxton \& Hay, 2005).

In general, however, GPs are poor at detecting eating disorders, particularly bulimia nervosa (Stager Bursten, Gabel \& Brose, 1996) and GPs are less likely than other health professionals to screen for eating disorder (Maradiegue, Cecelic \& Bozzelli, 1996). Estimates from overseas research suggest that although the prevalence of bulimia nervosa among adolescents and young female adults is approximately $1-3 \%$, as many as one third of GPs report having never seen a bulimic patient (Stager Bursten et al., 1996).

\section{Detection and management of eating disorders in rural and regional areas}

There is a lack of data on the prevalence of eating disorders in rural and regional areas, and data on eating disorder prevalence was notably absent from both the adult and adolescent components of the National Survey of Mental Health and Well Being (Andrews, Henderson \& Hall, 2001; Sawyer, Kosky, Graetz et al., 2000). There is some evidence that bulimia nervosa is more prevalent in urban centres, whereas anorexia nervosa occurs with equal frequency in areas with different degrees of urbanisation (Hoek, 1991). However, this is thought to be partly due to the drift of rural young people to urban centres as bulimia nervosa has a higher average age of onset than anorexia nervosa.
Significant inequity exists for rural patients in access to specialist psychiatric services, with many patients needing to travel to access such care (Boyd, Aisbett, Francis et al., 2006; Dixon \& Welch, 2000). As such, the GP has an integral role in obtaining early intervention and ensuring continuity of care for rural patients. In rural areas where specialist eating disorder services do not exist there are higher levels of ambulatory service use by these patients as well as higher numbers of medical admissions (Yuen, Gerdes \& Gonzales, 1996). Consultative linkages between mental health services and primary care practitioners are crucial in rural areas where there are no specialist eating disorder services.

\section{The present study}

The aim of our study was to explore the prevalence of eating disorders in primary care in the Ballarat region using data collected by the Ballarat and District Division of General Practice, and to highlight the role of GPs in the detection of management of eating disorders in regional Australia.

\section{Method}

\section{Participants}

Participants for this study were 36 general practitioners in the membership of the Ballarat and District Division of General Practice (B\&DDGP) in regional Victoria.

The B\&DDGP covers a geographical area of 7,300 square kilometres and includes the regional city of Ballarat as well as the towns of Daylesford, Creswick, Clunes, Skipton and Ballan. This area represents approximately 100 general practitioners meeting the health needs of a catchment population of approximately 122,000 people. The nearest specialist eating disorders service to Ballarat is the Eating Disorders Unit at the Royal Melbourne Hospital which is located approximately 110 kilometres from Ballarat's centre.

At the time of data collection (August 2005), B\&DDGP membership comprised 102 GPs; thus, the participation rate for this study was $35.3 \%$. Although modest, this response rate is typical for research involving regional GPs and parallels that of other published studies (e.g., Caldwell, Jorm, Knox et al., 2004). 


\section{Procedure}

In our study, we used anonymous data previously collated by the B\&DDGP on the prevalence of eating disorders and patterns of referral of eating disorder patients among GPs in their Division. These data were collected in August 2005 via a one page fax-back survey. The survey asked GPs to self report the frequency of presentation of eating disorders in their practice over the past 12 months. Information was also sought regarding their decision to either refer patients suffering from eating disorders on to more specialist services or to continue to treat the patient within their practice. GPs were asked to indicate how many patients they had referred for a hospital admission in the past 12 months as well as the hospital to which these patients were admitted.

The anonymous data were coded and entered into the Statistical Package for the Social Sciences, Version 14.0 for analysis.

\section{Results}

Thirty-five GPs indicated that they had seen patients with eating disorders over the 12 months prior to August 2005. Of these, 22 (62.9\%) indicated that they had seen less than 6 patients; $6(17.1 \%)$ indicated that they had seen $6-10$ patients; 3 (8.6\%) indicated that they had seen 11-15 patients; 1 (2.8\%) indicated seeing 16-20 patients; and $3(8.6 \%)$ indicated that they had seen more than 20 patients in the past year. Only one GP had seen no eating disorder patients in the 12 months preceding the survey.

Of the GPs who had seen eating disorders in the 12 month period, 17 (48.5\%) indicated that bulimia nervosa was the most common form of eating disorder present in their practices. Anorexia nervosa was the most common form of eating disorder, present in $34.2 \%$ of cases, and other forms of eating disorders such as overeating were most common for $17.1 \%$ of GPs.

Of the GPs who had seen eating disorder patients, 18 (51.4\%) indicated that they would treat patients in their practices whereas 16 (48.6\%) indicated that they would refer eating disorder patients to other services. Referral patterns are summarised in Table 1. GPs referred more often to mental health professionals (i.e., psychiatrists, psychologists) and dietitians than to generic counselling services or community health services. Referrals to mental health professionals and dietitians constituted $65 \%$ of all referrals made. Only one GP had referred to the Primary Mental Health Team.

Exploration of these data suggested a trend whereby GPs who saw more patients in the past 12 months appeared more likely to treat patients in their practices than to refer them on. This trend is illustrated in Figure 1. A chi-square analysis revealed that GPs who saw more than 15 patients with eating disorders over the previous 12 months were significantly less likely to refer them to other services and more likely to treat them $\left[\chi^{2}(1)=4.27, p=.04\right]$. However, subsequent analyses revealed these same GPs tended to be less likely to have seen patients with clinical eating disorders, with this trend approaching statistical significance $\left[\chi^{2}(1)=\right.$ $3.10, \mathrm{p}=.08]$.

A relatively high proportion of GPs (22.9\%) had referred patients for an inpatient admission in the 12 months preceding the survey. Notably, the majority of these referrals $(62.5 \%)$ were to specialist eating disorder units in Melbourne and not to local hospitals.

Table 1. Referral patterns of regional GPs for patients with eating disorders

\begin{tabular}{lrr}
\hline $\begin{array}{l}\text { Professional to whom } \\
\text { referral was made }\end{array}$ & $\begin{array}{c}\text { GPs referring } \\
\text { to this source }\end{array}$ & $\begin{array}{r}\text { Percentage of } \\
\text { referrals }\end{array}$ \\
\hline Dietitian & 10 & 21.6 \\
Private Psychologist & 10 & 21.6 \\
Psychiatrist & 4 & 21.6 \\
Public Specialist Clinic & 3 & 8.6 \\
Community Health & & 6.4 \\
Counsellor & 3 & 6.4 \\
Paediatrician & 2 & 6.4 \\
Private Specialist Clinic & 1 & 2.1 \\
Eating Disorders Foundation & 1 & \\
of Victoria & 1 & 2.1 \\
Community Psychology Clinic & 1 & 2.1 \\
Primary Mental Health Team & & \\
University of Ballarat & 1 & \\
\hline
\end{tabular}

Note: GPs may have indicated that they referred to more than one source 


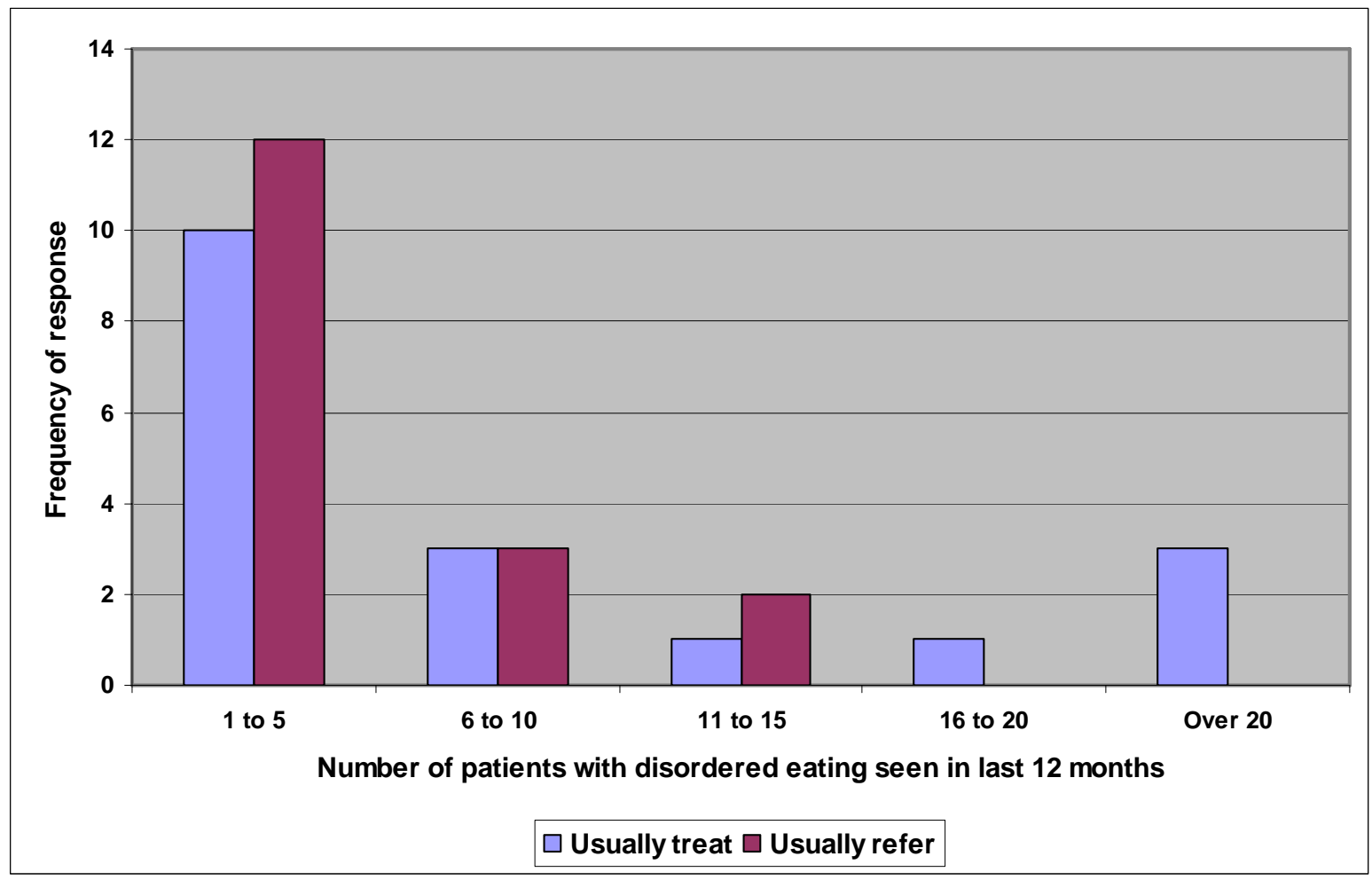

Figure 1. Treatment and referral patterns for participating GPs over a 12-month period

\section{Discussion}

Taking into account that the respondents to this survey represent one third of the total Division's membership, the results of this study suggest that GPs in regional Australia are successfully detecting eating disorders in their patients when compared to previously reported international rates (Hoek, 1991).

When comparing referral patterns with overseas data, results of this study suggest that regional GPs in Australia are much more likely to treat eating disorders within their practices than GPs in other countries (Stager Bursten et al., 1996). When these GPs do refer their patients, they are referring to mental health professionals, and they are significantly more likely to do this if the patient is suffering from a clinical eating disorder. This suggests that GPs are referring to specialists with expertise in the treatment of eating disorder in the absence of a publiclyfunded specialist service in the region.

One of the more notable findings of this study was that regional GPs are referring their patients for admission to specialist public and private eating disorder units in Melbourne rather than admitting them to one of two local hospitals (one public and one private). This highlights the rural health inequities that have been previously documented in the literature (Boyd et al., 2006; Dixon \& Welch, 2000). There are significant implications for patients from rural and regional centres and their families when they are referred to metropolitan centres for inpatient treatment, particularly when inpatient admissions for eating disorder may be for as long as four months for the first admission. This in turn reduces the likelihood of early intervention and worsens the prognosis for the patient (Steinhausen et al., 2003).

\section{Methodological issues}

A major shortcoming of this study is that we do not know the patterns for GPs who did not respond to the survey and that it is likely that the GPs who were motivated to respond were those who were seeing eating disorder patients. Another limitation is that we can only speculate about the reasons behind these referral patterns. Additional interview-based research with GPs is needed to achieve greater understanding of the GPs role and the rationale behind decisions to treat or not to treat these patients. 
Another limitation of this study is that it was not possible to confirm the presence of disordered eating in the patients referred on to services by GPs. Often patients are referred on quickly and without formal diagnostic evaluation, and as such we cannot know what proportion of the patients referred met diagnostic criteria for an eating disorder. We have made the assumption that GPs within the sample are highly trained and capable of accurately diagnosing patients presenting with eating disorder-related symptoms, and that this is done in a consistent fashion between GPs and between medical practices. There is evidence from a recent Australian study to support this assumption (see Hay, deAngelis, Millar \& Mond, 2006).

\section{Conclusions and recommendations}

This study highlights the work GPs do to treat eating disorders in the context of a regional community. Findings suggest that in the absence of local specialist services, the burden of care of these patients falls to the GP. GPs in this study were less likely to refer their patients to other services for treatment, highlighting the limited access to specialist treatment for patients in some regional cities in Australia. There is a need to recognise and support regional GPs and the work they do to detect and manage patients with eating disorders, within the limits of what is reasonable to expect of them in the context of a busy regional practice.

Based on the results of this research, we recommend the establishment of linkage partnerships between GPs and other service providers to address the lack of a specialist service in regional areas. There is also a strong case for conducting an independent analysis of the community's need for a specialist eating disorder service in the Ballarat region to which GPs can refer more severe cases requiring specialist treatment. There is potentially a need for upskilling of the regional GPs who currently provide treatment services to their eating disorder patients; however, there is first a need for further research to determine the current treatment practices of these GPs.

\section{Acknowledgements}

Thanks to Narelle Rinaldi who collected the data for this study and to the GPs from the Ballarat and District Division of General Practice who took part. Andrew Howard was CEO of the Ballarat and District
Division of General Practice at the time of this study. Correspondence with the Division regarding this study should be directed to the new CEO, Mr Andrew McPherson, at AndrewM@bddgp.org.au

\section{References}

Andrews, G., Henderson, S., \& Hall, W. (2001). Prevalence, comorbidity, disability and service utilization: Overview of the Australian national mental health survey. British Journal of Psychiatry, 178, 145-153.

Banasiak, S., Paxton, S., \& Hay, P. (2005). Guided self-help for bulimia nervosa in primary care: a randomized controlled trial. Psychological Medicine, 35, 1283-1294.

Boyd, C.P., Aisbett, D.L., Francis, K., Kelly, M., Newnham, K., \& Newnham, K. (2006). Issues in rural adolescent mental health in Australia. Rural and Remote Health, 6 (online), no. 501. http://www.rrh.org.au/articles/subviewnew.asp?Articl $\underline{\mathrm{eID}=501}$

Caldwell, T.M., Jorm, A.F., Knox, S., Braddock, D., Dear, K.B.G., \& Britt, H. (2004). General practice encounters for psychological problems in rural, remote, and metropolitan areas of Australia. Australian and New Zealand Journal of Psychiatry, 38, 774-780.

Dixon, J. \& Welch, N. (2000). Researching the ruralmetropolitan health differential using the social determinants of health. Australian Journal of Rural Health, 8, 254-260.

Hall, A., Slim, E., \& Hawker, F. (1984). Anorexia nervosa: long term outcome in 50 female patients. British Journal of Psychiatry, 145, 407-413.

Hay, P.J., de Angelis, C., Millar, H., \& Mond, J. (2006). Bulimia nervosa literacy of general practitioners. Primary Care and Community Psychiatry, 3, 103-108.

Hay, P.J., Marley, J., \& Lemar, S. (1998). Covert eating disorders: Prevalence, characteristics, and helpseeking of those with bulimic eating disorders in general practice. Primary Care Psychiatry, 4, 95-99.

Hoek, H. (1991). The incidence and prevalence of anorexia nervosa and bulimia nervosa in primary care. Psychological Medicine, 21, 455-460.

Maradiegue, A., Cecelic, E., \& Bozzelli, M. (1996). Do primary care providers screen for eating disorders? Gastroenterology Nursing, 19, 1807-1813.

Mehler, P. (2001). Diagnosis and care of patients with anorexia nervosa in primary care settings. Annals of Internal Medicine, 134, 1048-1059. 
Ogg, E., Millar, H., \& Pusztai, E. (1997). General practice consultation pattern preceding diagnosis of eating disorders. International Journal of Eating Disorders, 22, 89-93.

Sawyer, M., Kosky, R., Graetz, B., Arney, F., Zubrick, S.R., \& Baghurst, P. (2000). The National Survey of Mental Health and Wellbeing: the child and adolescent component. Australian and New Zealand Journal of Psychiatry, 34, 214-220.

Sayag, S. \& Latzer, Y. (2002). The role of the family physician in eating disorders. International Journal of Adolescent Medicine and Health, 14, 261-267.
Stager Bursten, M., Gabel, L., \& Brose, J. (1996). Detecting and treating bulimia nervosa: How involved are family physicians? Journal of the American Board of Family Medicine, 9, 241-248.

Steinhausen, H., Rauss-Mason, C., \& Seidel, R. (2003). Follow up studies of anorexia nervosa: A review of four decades of outcome research. Psychological Medicine, 21, 447-454.

Yuen, E., Gerdes, J., \& Gonzales, J. (1996). Patterns of rural mental health care: An exploratory study, General Hospital Psychiatry, 18, 14-21. 